\title{
Does Saving Uterus Save Ovaries?
}

\author{
Suvarna Khadilkar ${ }^{1}$
}

Published online: 18 March 2020

(c) Federation of Obstetric \& Gynecological Societies of India 2020

\begin{abstract}
Ovarian function after hysterectomy is a subject of much controversy since many years. There is increasing awareness among gynecologists that the ovaries need to be spared at hysterectomy for benign conditions. However the awareness is limited, and many practitioners believe in removal of ovaries at hysterectomy. Removal of ovaries will save lives of patients at risk of developing ovarian cancer. But it will be unnecessary for low-risk women and will in fact endanger their lives by increasing cardiac risks. Recent data suggest that ovarian epithelial cancer arises from tubal epithelium, which adds new option of performing only salpingectomy and sparing ovaries, which will serve dual purpose of preventing ovarian cancer and continuing productive function of ovaries. Reproductive function is lost with hysterectomy. But it may be worth looking at impact of hysterectomy on productive function of ovaries. This editorial will focus on this issue and look at evidence on effects of other gynecological procedures on ovarian function.
\end{abstract}

\section{Introduction}

Ovarian function after hysterectomy has been studied by several authors since many years, but we are yet to reach a consensus on this issue. It is recommended that healthy ovaries be routinely spared at the time of hysterectomy, except for high-risk women in whom prophylactic oophorectomy is advocated. Ovaries perform two basic functions; one is reproduction and the other is production of hormones, the endocrine function. Ovaries continue to secrete androgens beyond menopause up to 65 years [1], and these are converted into estrogens peripherally, in amounts sufficient to maintain bone, cardiac and urogenital health for up to 10 years beyond menopause. [2].

It is interesting to know what happens to the function of these ovaries conserved at hysterectomy. Do they result in early menopause? Hysterectomy brings about gradual loss

Prof. Suvarna Satish Khadilkar MD DGO FICOG, CIMP, Diploma in Endocrinology (UK), is Editor-in-Chief of Journal of Obstetrics and Gynecology of India and Treasurer of FOGSI; she is Professor and Head, and Consultant Gyne-Endocrinologist at Bombay Hospital and Medical Research Centre, Mumbai, Former Professor and Head at the Dept of Ob-Gyn, RCSM, Government Medical College, Maharashtra, and Associate Professor and Unit Chief Grant Medical College and Cama and Albless Hospital, Mumbai.

Suvarna Khadilkar

suvarnakhadilkar2@gmail.com

1 Mumbai, India of ovarian estrogen production over 2-3 years, but the androgenic secretion of conserved ovaries continues till another 10 years. However, these women will still suffer from complete estrogen deficiency much earlier, when compared with women with intact uteri.

Assessment of ovarian function is done in several ways. Reproductive potential is assessed by measuring the antral follicles count (AFC), serum anti-mullerian hormone (AMH) and serum inhibin B levels. Serum estradiol levels, serum follicular stimulating hormone levels, vascularity of ovaries on doppler, ovarian volume and endometrial thickness on ultrasound will indicate endocrine function of ovaries. Such comprehensive assessment is desirable but has not been reported in many studies. Studies also have other pitfalls such as retrospective designs, lack of a control group, symptomatology not supported by laboratory values, small sample sizes or short-term follow-ups. This editorial will critically analyze available literature and seek way forward.

\section{Hysterectomy and Ovarian Function}

The exact mechanism by which hysterectomy affects ovarian function is ill understood. One assumption is that hysterectomy brings about increased rate of follicular atresia, leading to premature menopause. Thrombosis of ovarian blood vessels with a subsequent reduction in ovarian blood supply is another hypothesis [3]. Whether it is the indication or the 
procedure of hysterectomy responsible for ovarian dysfunction is unclear. It may be hypothesized that if the target organ is removed, the endocrine function ceases slowly.

It is a clinical observation that women develop symptoms of menopause after couple of years of ovary-sparing premenopausal hysterectomy. However, we do understand that experience and evidence may not go hand in hand. Literature on impact of premenopausal hysterectomy on the ovarian function is divided. Some research indicates an increase in the incidence and severity of menopausal symptoms and ovarian failure following the removal of the uterus, despite the ovaries remaining in place. On the other hand, some researchers report no decrease in ovarian function [4]. Bhattacharya and colleagues reported that in comparison with endometrial ablation, simple hysterectomy for dysfunctional uterine bleeding does not compromise bladder or ovarian function. The authors assessed ovarian function over 2 years by measuring FSH levels and performing symptoms analysis in both groups. However, in spite of these results, they expressed the need for caution in concluding that hysterectomy has no effect on ovarian function.

More recent data suggest that there is no significant difference in ovarian function after premenopausal hysterectomy up to 1 year. Abdelzeim and colleagues studied ovarian blood supply by Doppler and concluded that the ovarian pulsatile index (PI) reduced and ovarian volume increased indicating increased blood supply and documented maintained AMH, FSH and estradiol levels at 1-year follow-up period [5]. Nahás et al. also observed reduced PI values on Doppler ultrasonography suggesting decrease in the resistance flow in the ovarian arteries in women of reproductive age who underwent total abdominal hysterectomy [6].

There is a body of research which suggests that the ovarian function is adversely affected by hysterectomy. Derksen and colleagues reported that there is a significant difference in FSH levels 2 years after simple hysterectomy [7]. Ahn et al. [8] reported that women develop symptoms of menopause within $1.99 \pm 2.40$ years after the premenopausal hysterectomy. Trabuco et al. [9] observed substantial decrease in AMH levels at 1 year, while Nahas et al. [10] observed reduced inhibin B levels up to 1 year. Farquhar and colleagues [11] estimated that menopause occurred nearly 4 years earlier in women undergoing hysterectomy as compared to women who did not have the surgery. Moorman and colleagues [12] reported twofold increased risk of ovarian failure among women undergoing ovary-sparing hysterectomy. As observed, there is no uniformity in selection of tests of ovarian function in these studies. Whether ovarian failure affects the health and survival of women is most relevant information we need today. One such study reported that ovary-sparing hysterectomy is associated with an increased long-term risk of cardiovascular and metabolic conditions, especially in women who undergo hysterectomy at the age of $\leq 35$ years. Laughlin-Tommaso et al. [13] recommended conservation of the uterus to save ovarian function and reduce cardiometabolic risks.

So the issue seems equipoised in the literature; there are papers suggesting that the ovarian function is unaffected, but there are an equal number of papers suggesting that ovarian function is compromised after ovary-sparing premenopausal hysterectomy. The true magnitude of the ill effects of ovary-sparing hysterectomies is perhaps difficult to judge and may actually be more prevalent than what has been documented; as in the current practice pattern, patients with cardiac symptoms are likely to report to cardiologists and be tracked by them. Likewise patients with fractures also will always report to the orthopedicians rather than a gynecologists. The link between these sequelae and hysterectomy may go unrecognized and thus be undocumented.

\section{Does Type of Hysterectomy Affect the Ovarian Function?}

Various types of hysterectomy have been studied and compared by different authors. Total abdominal hysterectomy (TAH), laparoscopic hysterectomy, radical hysterectomy and subtotal hysterectomy have all shown varying degrees of ovarian function compromise.

Moorman et al. [12] reported that the risk of ovarian failure was higher for women who had a unilateral oophorectomy along with their hysterectomy as compared to women who had a hysterectomy with both ovaries left intact. One study compared subtotal hysterectomy by laparoscopy and laparotomy and found that laparoscopic subtotal hysterectomy is associated with lower rates of loss of ovarian function [14]. Risk of early menopause is lower in women undergoing subtotal hysterectomy compared to total hysterectomy. Ishii et al. [15] studied ovarian function after radical hysterectomy with ovarian preservation for cervical cancer. While cancer risk did not increase in this study, 15 of 33 patients became menopausal after treatment. There was a significant correlation with the age at radical hysterectomy. The younger the patient, the better was the ovarian function preservation.

Ovarian transposition is often used before radical hysterectomy and radiotherapy. Gubbala et al. [16] concluded in their review that ovarian transposition is associated with $90 \%$ preservation of ovarian function and negligible risk of metastases to the transposed ovaries despite common incidence of ovarian cysts. The preservation of ovarian function dropped to $65 \%$ when external radiation was used postoperatively. They encouraged its use for younger women to preserve ovarian function. 


\section{Does Salpingectomy Affect Ovarian Function?}

A randomized controlled trial by Asgari et al. [17] reported that prophylactic bilateral salpingectomy at the time of laparoscopic hysterectomy for risk reduction for cancer has no negative effect on ovarian reserve and does not increase the surgical risk. So salpingectomy is a better option to prevent cancer risk rather than sacrificing ovaries. Yuan et al. [18] concluded that hysterectomy with bilateral salpingectomy compromised ovarian reserve, with the damage being most severe among younger patients.

Venturella et al. [19], on the other hand, compared women who underwent ovary-sparing total laparoscopic hysterectomy and bilateral salpingectomy with women who had their uteri and tubes intact. They concluded that there is no difference in the ovarian function as assessed by FSH, AMH, doppler indices and AFC. Behnamfar et al. [20] concurred, showing similar results and recommending bilateral salpingectomy with hysterectomy for benign conditions. Song et al. [21] compared laparoscopic hysterectomy with and without opportunistic salpingectomy and concluded that additional salpingectomy does not increase the surgical risk and does not affect ovarian function adversely compared with the control group.

\section{Other Gynecological Procedures and Ovarian Function}

Uterine artery embolization (UAE) certainly has a risk of early ovarian failure. Hehenkamp et al. [22] reported that UAE and hysterectomy both affect ovarian reserve. However, majority of other studies have shown that there is no significant difference in ovarian function after the procedure.

Rashid et al. [23] studied the effects of UAE and surgical treatment on ovarian function in women with uterine fibroids and concluded that there is increased incidence of ovarian failure in women over 45 years of age after UAE as well. This effect was less pronounced in younger women.

Internal iliac ligation done to control obstetric hemorrhage is generally not followed by any serious sequel due to collateral circulation. In one interesting study, author conducted quantitative digital angiography and angio$\mathrm{CT}$ of the ovarian arteries and the ovarian branches of the uterine arteries from 6 to 26 months after the internal iliac ligation operation [24]. Serum AMH and FSH were assessed on day 3 of cycle. Reversal of blood flow in the ovarian branches of the uterine arteries was observed. They also observed that mean ovarian artery diameter was significantly dilated, and AMH levels were significantly lower compared to control group [24]. Although this is a very good study, sample size was small. Therefore more studies are necessary to support these findings.

\section{Conclusion}

There is conflicting evidence on post-hysterectomy preservation of ovarian function. Since there are an enough number of papers which conclude that there is a reduction in ovarian function after simple hysterectomy, clinical experience seems to have substantial literature support. Then, should not we save uterus to save ovaries? We must keep in mind that there are a lot of procedures which we undertake routinely, may have a long-term impact on health of the women. Early menopause is probably one such aftermath of hysterectomy. Well-designed prospective studies with long-term multispeciality follow-ups are necessary to support these clinical findings. Till such robust data become available, we should follow the principle of judicious use of conservative line of management over more radical forms of treatment!

Saving uterus saves ovaries, saving ovaries saves lives!

\section{References}

1. Davison SL, Bell R, Donath S, et al. Androgen levels in adult females: changes with age, menopause, and oophorectomy. J Clin Endocrinol Metab. 2005;90(7):3847-53.

2. Rocca WA, Grossardt BR, De Andrade M, et al. Survival patterns after oophorectomy in premenopausal women: a population-based cohort study. Lancet Oncol. 2006;7(10):821-8.

3. Deng CY, Tang DM, Yu Q, et al. Effect of premenopausal hysterectomy on ovarian function. Zhongguo Yi Xue Ke Xue Yuan Xue Bao. 2002;24:639-42.

4. Bhattacharya S, Mollison J, Pinion S, et al. A comparison of bladder and ovarian function two years following hysterectomy or endometrial ablation. Br J Obstet Gynaecol. 1996;103:898-903.

5. Abdelazim IA, Abdelrazak KM, Elbiaa AA, et al. Ovarian function and ovarian blood supply following premenopausal abdominal hysterectomy. Przeglad menopauzalny=Menopause Review. 2015;14(4):238.

6. Nahás EA, Pontes A, Nahas-Neto J, et al. Effect of total abdominal hysterectomy on ovarian blood supply in women of reproductive age. J Ultrasound Med. 2005;24(2):169-74.

7. Derksen JG, Brölmann HA, Wiegerinck MA, et al. The effect of hysterectomy and endometrial ablation on follicle stimulating hormone (FSH) levels up to 1 year after surgery. Maturitas. 1998;29(2):133-8.

8. Ahn EH, Bai SW, Song CH, et al. Effect of premenopausal hysterectomy on ovarian function. Yoseni Med J. 2002;43:53-8.

9. Trabuco EC, Moorman PG, Algeciras-Schimnich A, et al. Association of ovary-sparing hysterectomy with ovarian reserve. Obstet Gynecol. 2016;127(5):819.

10. Nahas E, Pontes A, Traiman P, et al. Inhibin B and ovarian function after total abdominal hysterectomy in women of reproductive age. Gynecol Endocrinol. 2003;17(2):125-31. 
11. Farquhar CM, Sadler L, Harvey SA, et al. The association of hysterectomy and menopause: a prospective cohort study. BJOG. 2005;112(7):956-62.

12. Moorman PG, Myers ER, Schildkraut JM, et al. Effect of hysterectomy with ovarian preservation on ovarian function. Obstet Gynecol. 2011;118(6):1271.

13. Laughlin-Tommaso SK, Khan Z, Weaver AL, et al. Cardiovascular and metabolic morbidity after hysterectomy with ovarian conservation: a cohort study. Menopause (NY). 2018;25(5):483.

14. Li M, Zhou Li-h, Han X, et al. The study of the effect of ovarian function under subtotal abdominal hysterectomy and subtotal laparoscopic hysterectomy. http://en.cnki.com.cn/Article_en/CJFDT otal-HLYX201003009.htm. 2010-03.

15. Ishii K, Aoki Y, Takakuwa K, et al. Ovarian function after radical hysterectomy with ovarian preservation for cervical cancer. J Reprod Med. 2001;46:347-52.

16. Gubbala K, Laios A, Gallos I, et al. Outcomes of ovarian transposition in gynaecological cancers; a systematic review and metaanalysis. J Ovarian Res. 2014;7(1):69.

17. Asgari Z, Tehranian A, Rouholamin S, et al. Comparing surgical outcome and ovarian reserve after laparoscopic hysterectomy between two methods of with and without prophylactic bilateral salpingectomy: a randomized controlled trial. J Cancer Res Ther. 2018;14(3):543.

18. Yuan Z, Cao D, Bi X, et al. The effects of hysterectomy with bilateral salpingectomy on ovarian reserve. Int J Gynecol Obstet. 2019;145(2):233-8.

19. Venturella R, Lico D, Borelli M, et al. 3 to 5 years later: long-term effects of prophylactic bilateral salpingectomy on ovarian function. J Minim Invasive Gynecol. 2017;24(1):145-50.

20. Behnamfar F, Jabbari H. Evaluation of ovarian function after hysterectomy with or without salpingectomy: a feasible study. J Res Med Sci Off J Isfahan Univ Med Sci. 2017;22:68.

21. Song T, Kim MK, Kim ML, et al. Impact of opportunistic salpingectomy on anti-Müllerian hormone in patients undergoing laparoscopic hysterectomy: a multicentre randomised controlled trial. BJOG: Int J Obstet Gynaecol. 2017;124(2):314-20.

22. Hehenkamp WJ, Volkers NA, Broekmans FJ, et al. Loss of ovarian reserve after uterine artery embolization: a randomized comparison with hysterectomy. Hum Reprod. 2007;22(7):1996-2005. https://doi.org/10.1093/humrep/dem105 PMID: 17582145.

23. Rashid S, Khaund A, Murray LS, et al. The effects of uterine artery embolisation and surgical treatment on ovarian function in women with uterine fibroids. BJOG. 2010;117(8):985-9.
24. Raba G. Effect of internal iliac artery ligation on ovarian blood supply and ovarian reserve. Climacteric. 2011;14(1):54-7.

Publisher's Note Springer Nature remains neutral with regard to jurisdictional claims in published maps and institutional affiliations.

\section{About the Author}

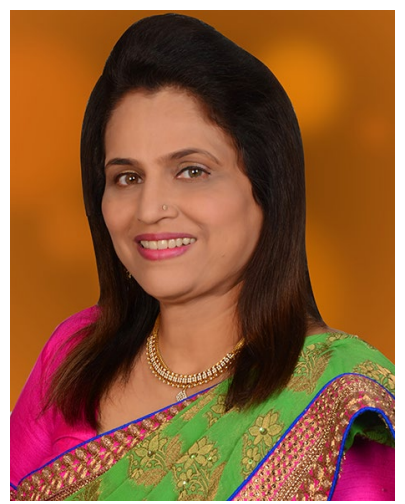

Dr Suvarna Khadilkar is the Editor-in-Chief of this journal. She is currently Professor and Head of the Department of Obstetrics and Gynecology and Consultant Gyne-Endocrinologist at Bombay Hospital Institute of Medical Sciences and Medical Research Centre, Mumbai. In the past, she worked as an Associate Professor and Unit Chief at JJ Group of Hospitals and Grant Medical College (GMC), Mumbai and thereafter in the capacity of the Professor and Head in Department of Obstetrics and Gynecology, Government Medical College, Kolhapur, Maharashtra. She has over 30 years of teaching experience as an undergraduate and postgraduate teacher and examiner at Mumbai University and Maharashtra University of Health Sciences. Pursuing her interest in endocrinology, she acquired Diploma in Endocrinology from the prestigious University of South Wales, UK, and has been appointed as a recognized teacher in endocrinology in University of South Wales. She is currently the Treasurer of FOGSI, Treasurer of Mumbai Ob-Gyn Society and Treasurer of Association of Medical Women in India (AMWI). She has held many prestigious positions like President of Indian Menopause Society, Chairperson of Reproductive Endocrinology Committee of FOGSI, President of AMWI, Mumbai. She has published six textbooks and more than 70 articles at national and international levels. She is a recipient of 30 awards for her research work including the Young Scientist Award. 